Pesq. Vet. Bras. 37(12):1423-1429, dezembro 2017 DOI: $10.1590 / \mathrm{S} 0100-736 \mathrm{X} 2017001200011$

\title{
Aspectos epidemiológicos, clínico-patológicos e diagnóstico precoce da seneciose bovina no Oeste do Rio Grande do Sul ${ }^{1}$
}

\author{
Adriano A. Krabbe ${ }^{2}$, Maiara A. Gonçalves ${ }^{3}$, Caroline S. Silveira ${ }^{2}$, Raissa M. Morais ${ }^{3}$, \\ Lucas S. Quevedo ${ }^{3}$, Paula V.R. Ribeiro ${ }^{4}$ e Bruno L. Anjos ${ }^{3 *}$
}

\begin{abstract}
Krabbe A.A., Gonçalves M.A., Silveira C.S., Morais R.M., Quevedo L.S., Ribeiro P.V.R. \& Anjos B.L. 2017. [Epidemiological, clinical and pathological aspects and early diagnosis of bovine seneciosis in the Western region of Rio Grande do Sul, Brazil.] Aspectos epidemiológicos, clínico-patológicos e diagnóstico precoce da seneciose bovina no Oeste do Rio Grande do Sul. Pesquisa Veterinária Brasileira 37(12):1423-1429. Laboratório de Patologia Veterinária, Universidade Federal do Pampa, Uruguaiana, RS 97500-970, Brazil. E-mail:anjosbl@gmail.com

The ingestion of Senecio spp. (ragwort) is perhaps the leading cause of death of cattle in central and southern Rio Grande do Sul, Brazil. Considering the limited information about this condition in the Western region of Rio Grande do Sul, Brazil, this paper describes main epidemiological clinical and pathological aspects of seneciosis in cattle. The assessments were made through questionnaires on 16 rural properties which had suspected cases of poisoning in cattle, from August 2011 to March 2014. During the visits were evaluated epidemiological aspects of poisoning and performed sample collection of Senecio plants for botanical identification, as well as collection of liver samples using transthoracic biopsy for confirmation of the poisoning. Senecio species most common on farms were S. brasiliensis and $S$. heterotrichius. From 88 cattle that failed to thrive on nine different farms, 69 animals (about $80 \%$ ) were positive for chronic seneciosis with periportal fibrosis (78\% of cases), megalocytosis ( $76 \%$ of cases) and with bile duct proliferation ( $68 \%$ of cases). Lesions were classified as mild, moderate or marked. The current study confirms the occurrence of this poisoning, even in cattle without evident clinical signs. The use of liver biopsy enabled the early diagnosis of poisoning and helped farmers carefully to dispose affectd cattle, as well as to recognize the real situation of poisoning in the herd and minimize economic losses.
\end{abstract}

INDEX TERMS: Poisonous plants, diseases of cattle, chronic hepatopathy, pyrrolizidine alkaloids, plant poisoning, pathology, toxicoses.

RESUMO.- A ingestão de Senecio spp. (maria-mole) é, possivelmente, a principal causa de morte de bovinos por agentes tóxicos nas regiões central e sul do Rio Grande do Sul. Ao considerar a limitação de informações acerca dessa condição no Oeste do Rio Grande do Sul, esse trabalho ob-

\footnotetext{
${ }^{1}$ Recebido em 19 de maio de 2017.

Aceito para publicação em 8 de junho de 2017.

Parte da Dissertação de Mestrado do primeiro autor.

${ }^{2}$ Programa de Pós-Graduação em Ciência Animal, área de concentração em Sanidade Animal/Patologia Veterinária, Universidade Federal do Pampa (Unipampa), Uruguaiana, RS 97500970, Brasil.

${ }^{3}$ Laboratório de Patologia Veterinária (LPV), Hospital Universitário Veterinário, Unipampa, Uruguaiana, RS 97500970, Brasil. *Autor para correspondência: anjosbl@gmail.com.br

${ }^{4}$ Médica Veterinária da Inspetoria Veterinária do Rio Grande do Sul, Maçambará, RS 97645000, Brasil.
}

jetiva descrever os principais aspectos epidemiológicos e clínico-patológicos da seneciose em bovinos nessa região. 0 estudo foi realizado por meio da aplicação de questionários em 16 propriedades rurais de municípios da região que apresentaram casos suspeitos da intoxicação em bovinos, de agosto de 2011 a março de 2014. Durante as visitas as propriedades foram coletadas plantas do gênero Senecio para identificação botânica, bem como se procedeu a coleta de fragmentos de fígado através de biópsia transtorácica para confirmação da intoxicação. As espécies de Senecio mais frequentes nas propriedades foram $S$. brasiliensis e $S$. heterotrichius. De um total de 88 bovinos, de nove propriedades distintas, que apresentaram falha no ganho de peso, 69 animais (aproximadamente 80\%) foram positivos para seneciose crônica por apresentarem lesões características da intoxicação, tais como fibrose periportal (78\% 
dos casos), megalocitose (76\% dos casos) e proliferação de ductos biliares (68\% dos casos), classificadas entre discretas e acentuadas. 0 trabalho confirmou a ocorrência da doença, mesmo em bovinos sem sinais clínicos evidentes. Os resultados obtidos nessas avaliações foram fundamentais para orientar proprietários e técnicos quanto às principais características da doença e às formas de controle a serem adotadas. 0 emprego da biópsia hepática possibilitou o diagnóstico precoce da intoxicação e auxiliou os criadores quanto ao descarte mais criterioso de bovinos e a real situação da intoxicação no rebanho, minimizando as perdas econômicas.

TERMOS DE INDEXAC ÃO: Plantas tóxicas, doenças de bovinos, hepatopatia crônica, alcaloides pirrolizidínicos, intoxicação por planta, patologia, toxicoses.

\section{INTRODUÇÃO}

Plantas tóxicas de diversos gêneros são causadoras de prejuízos econômicos por induzirem elevada mortalidade de bovinos no Brasil (Riet-Correa et al. 2007, Tokarnia et al. 2012, Pessoa et al. 2013). As plantas que causam alterações hepáticas e que têm como princípio tóxico os alcaloides pirrolizidínicos (AP) estão entre as principais (Anjos et al., 2010, Tokarnia et al. 2012). No Rio Grande do Sul, destacam-se as várias espécies de Senecio, que são responsáveis por uma alta mortalidade de bovinos no estado (Karam et al. 2004, Riet-Correa et al. 2007, Tokarnia et al. 2012).

As plantas do gênero Senecio (família Asteraceae) são conhecidas popularmente como maria-mole ou flor-das-almas. Essas plantas são anuais e encontram condições propícias de umidade, luminosidade, solo e temperatura para seu intenso desenvolvimento no Rio Grande do Sul (Karam et al. 2002). Atualmente, estima-se que mais de $10 \%$ das mortes de bovinos no Rio Grande do Sul sejam causadas por plantas tóxicas. Isso corresponde a uma média de 70 mil bovinos mortos por ano (Riet-Correa \& Medeiros 2001), e aproximadamente $50 \%$ destas mortes são ocasionadas pelas plantas do gênero Senecio (Riet-Correa \& Medeiros 2001, Schild et al. 2004, Rissi et al. 2007). Essa intoxicação por Senecio é a principal causa de morte de bovinos na região central (Rissi et al. 2007, Lucena et al. 2010) e sul (Karam et al. 2004) do Rio Grande do Sul. Os bovinos manifestam os sinais clínicos após dias ou meses de ingestão da planta, que progridem para a morte associada a cirrose hepática. Eventualmente, os sinais clínicos podem regredir por completo, mas os animais podem voltar a apresentá-los (Prakash et al. 1999).

Possivelmente, essa ocorrência seja ainda maior na região Oeste do Rio Grande do Sul, onde as características ambientais e métodos de manejo dos rebanhos favorecem a intoxicação. Embora o S. brasiliensis seja facilmente conhecido por técnicos e pecuaristas da região, nota-se grande dificuldade no reconhecimento de outras espécies de Senecio e que podem apresentar características morfológicas bastante distintas podendo levar a falhas no estabelecimento do diagnóstico da intoxicação (Anjos 2014).

Um dos métodos mais eficazes e práticos de diagnóstico da seneciose ainda é a avaliação histológica do fígado coletado no momento da necropsia ou através de biópsia hepática (Barros et al. 2007, Tokarnia et al. 2012), porém essa técnica ainda é pouco conhecida e praticada na região.

Considerando a importância da seneciose em bovinos no Rio Grande do Sul e a limitação de informações acerca dessa condição no Oeste do Rio Grande do Sul esse trabalho tem como objetivo descrever os principais aspectos epidemiológicos e clinicopatológicos da seneciose bovina na região e enfatizar a importância da biópsia hepática para o estabelecimento do diagnóstico precoce e conclusivo da intoxicação.

\section{MATERIAL E MÉTODOS}

O estudo ocorreu de agosto de 2011 a março de 2014 e avaliou propriedade rurais, predominantemente na região Oeste do Rio Grande do Sul com suspeita clínica de intoxicação crônica por Senecio spp. em bovinos. Foram analisados os aspectos epidemiológicos e clinico-patológicos da intoxicação através da aplicação de questionário padrão e avaliação in loco das propriedades. Os principais aspectos avaliados no estudo foram sumarizados no Quadro 1.

Sempre que solicitada foi realizada a coleta de fragmentos de fígado por biópsia hepática nos bovinos com algum tipo de atraso no desenvolvimento ou que apresentaram sinais de emagrecimento. 0 procedimento foi realizado com auxílio de agulha $155 \times 2 \mathrm{~mm}$ adaptada com êmbolo e abordagem transtorácica no penúltimo espaço intercostal direito, aproximadamente $20 \mathrm{~cm}$ abaixo da linha do dorso, após anestesia local com lidocaína a 2\% (Braga et al. 1985). Os fragmentos coletados foram fixados em formalina $10 \%$, identificados e processados rotineiramente para avaliação histopatológica através de cortes com $3 \mu \mathrm{m}$, corados por Hematoxilina e Eosina (H\&E) e Tricrômio de Masson. Foram considerados positivos para seneciose as amostras que apresentaram pelo menos duas das três principais alterações microscópicas observadas na intoxicação crônica por Senecio sp. em bovinos (Barros et al. 2007), sendo elas fibrose periportal, proliferação de ductos biliares e hepatomegalocitose, levando sempre em consideração a morfologia das lesões.

\section{RESULTADOS}

Foram investigadas 16 propriedades rurais pertencentes aos municípios de Maçambará ( 5 propriedades), Uruguaiana (4), São Borja (4), Quaraí (1), Alegrete (1), e Manoel Viana (1), as quais apresentaram mortalidade de bovinos com suspeita de intoxicação por Senecio. Os casos relatados ocorreram como casos isolados ou em forma de surtos, com morte de vários bovinos. 0 sistema de produção era extensivo e a forragem disponível consistia de campo nativo e grande quantidade de capim-annoni (Eragrostis plana). Das 16 propriedades avaliadas, 12 se caracterizavam por ciclo completo de produção (nascimento e terminação de bovinos), em três delas por terminação/engorda e pela criação de terneiros. Das 16 propriedades, 14 apresentaram a intoxicação sob a forma de surto, uma com um caso isolado (Propriedade 1) e em apenas uma delas não houve mortalidade (Propriedade 11). De um total geral de 12.328 bovinos sob risco de desenvolver a intoxicação, $310(2,51 \%)$ apresentaram sinais clínicos compatíveis com a doença e desses, $250(2,02 \%)$ morreram após apresentarem sinais clínicos e lesões macroscópicas compatíveis com a intoxicação crônica por Senecio spp. 


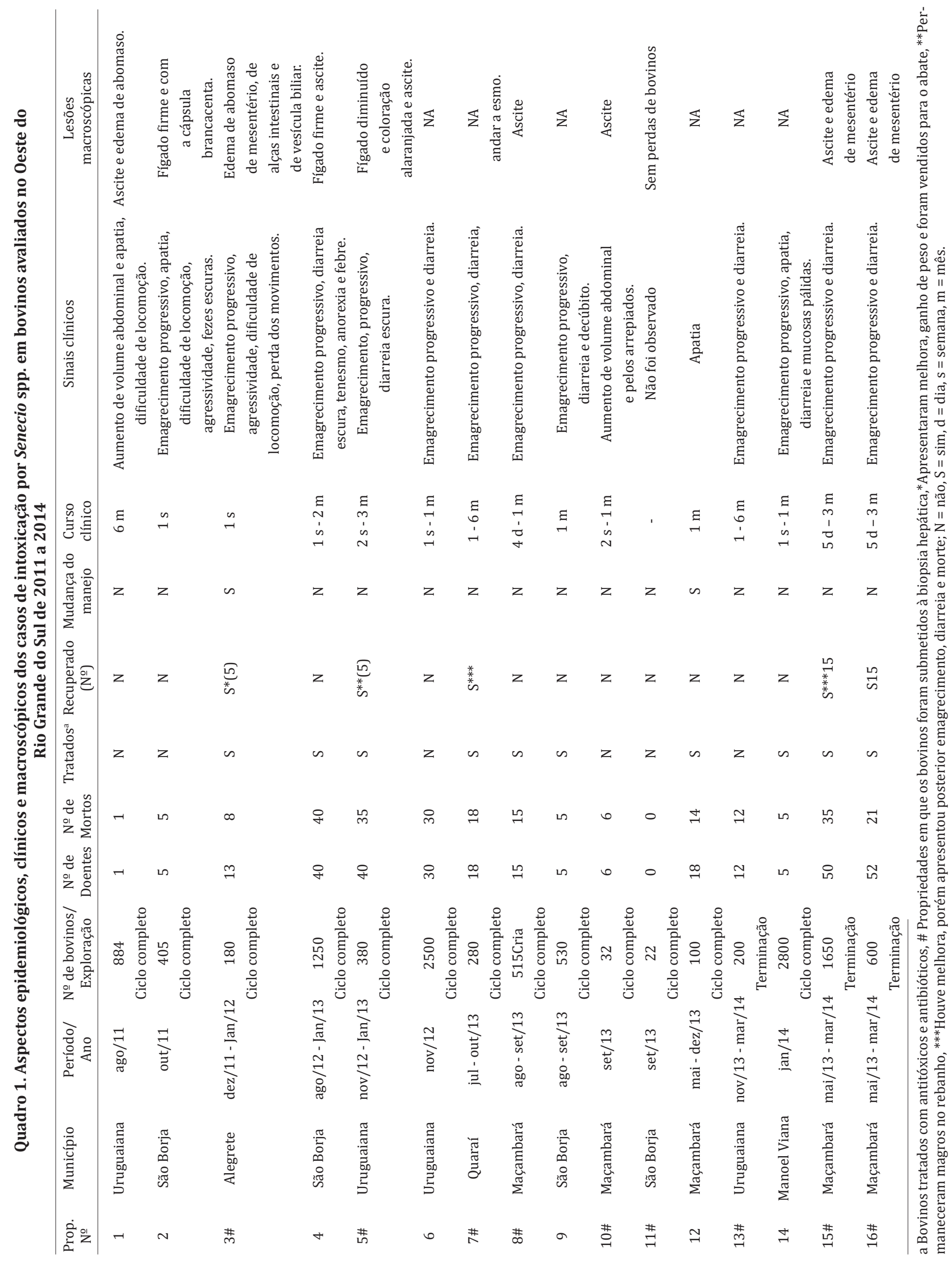




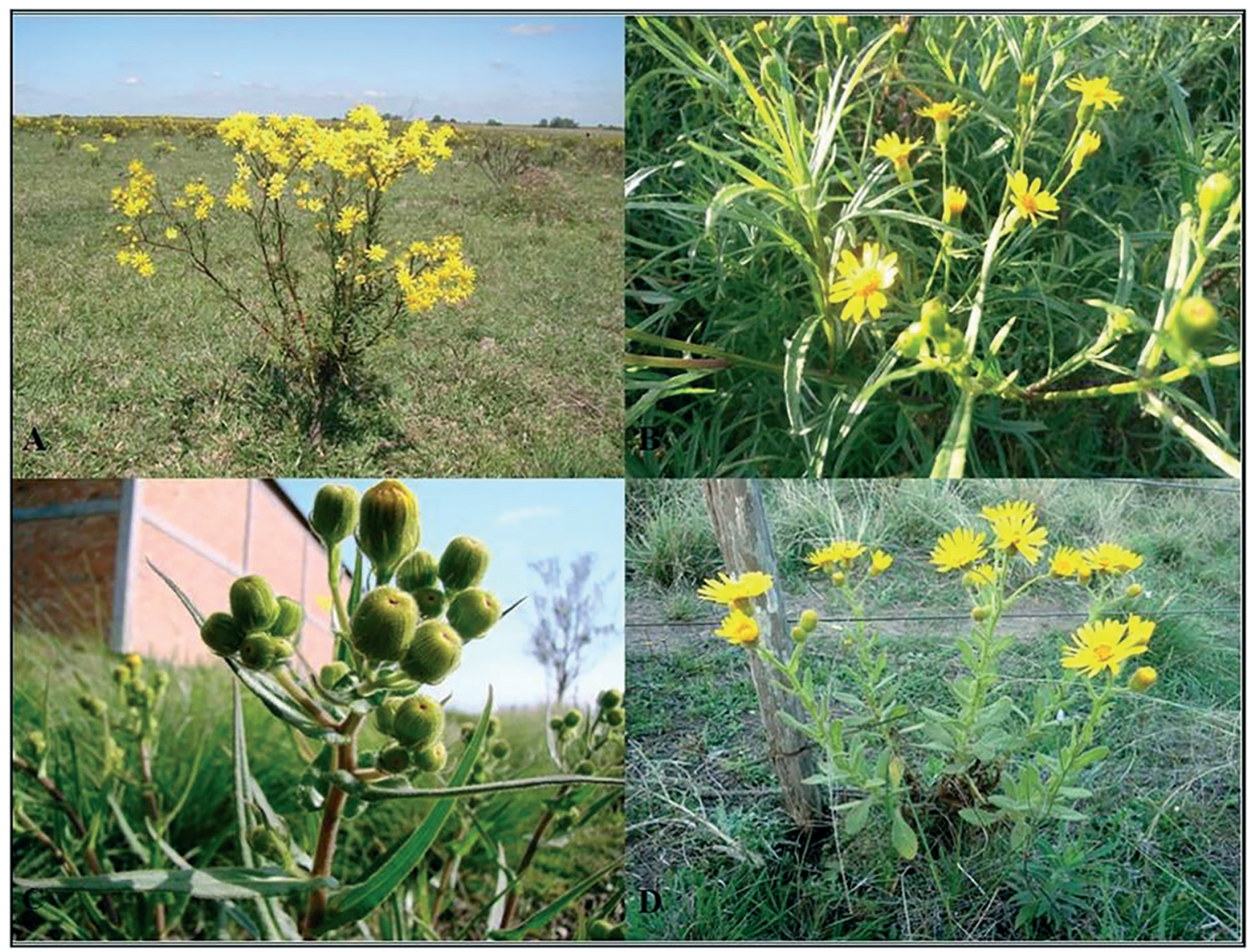

Fig.1. Exemplares das espécies de plantas do gênero Senecio em ordem de maior ocorrência nas propriedades estudadas. (A) Senecio brasiliensis, (B) Senecio heterotrichius, (C) Senecio oxyphyllus, (D) Senecio selloi.

Nas visitas técnicas entre os meses de maio e novembro dos anos de 2012 e de 2013 foram catalogadas diferentes espécies de Senecio spp., tais como: Senecio brasiliensis, $S$. heterotrichius, S. oxyphyllus, S. selloi (Fig.1) e S. leptolobus. Em todas as propriedades foram observadas pelo menos duas dessas espécies, com maior frequência do Senecio brasiliensis, presente em todas as propriedades. Senecio leptolobus foi encontrado apenas na Propriedade 7, no município de Quaraí-RS. Em nenhuma das propriedades eram utilizados métodos de controle da planta. Em algumas propriedades os ovinos eram criados apenas em pequeno número para consumo na propriedade.

Em 10 das propriedades $(3-5,7-9,12,14-16)$ foi instituído algum tipo de tratamento aos bovinos doentes, os quais se baseavam em medicamentos antitóxicos e antibióticos. Em apenas duas propriedades, os bovinos foram removidos das áreas invadidas por Senecio spp. Em cinco propriedades $(3,5,7,15,16)$ houve recuperação de bovinos intoxicados e em quatro dessas (3, 5, 7 e 15) alguns dos animais foram vendidos para o abate após melhora do estado geral e da condição corporal. Os animais que permaneceram magros na propriedade apresentaram novamente os sinais clínicos da intoxicação e morreram. Dessas cinco propriedades, todas apresentaram casos da intoxicação nas épocas que corresponderam ao final da primavera e ao verão, com exceção da Propriedade 7.

O curso clínico variou entre quatro dias e seis meses e caracterizou-se por emagrecimento progressivo, diarreia escura, apatia, dificuldade de locomoção e agressividade. Alguns bovinos foram necropsiados por médicos veterinários autônomos em nove propriedades e os principais

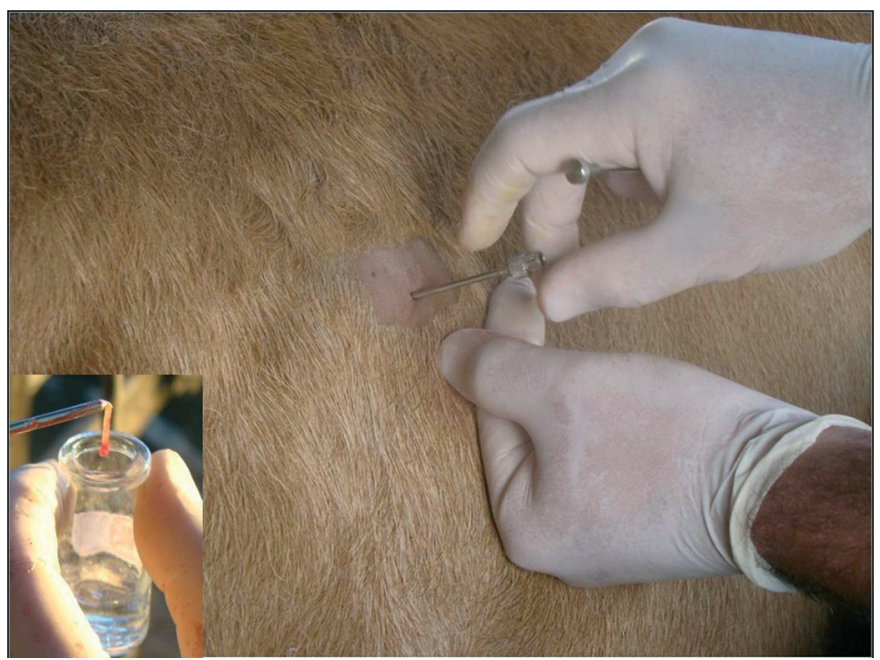

Fig.2. Realização de biópsia hepática em um bovino por acesso percutâneo e transtorácico no penúltimo espaço intercostal. No detalhe observa-se fragmento de fígado coletado com aproximadamente $1,5 \mathrm{~cm}$ de comprimento por $0,1 \mathrm{~cm}$ de espessura a ser acondicionado em frasco com formol a $10 \%$ para posterior avaliação histopatológica. 
achados macroscópicos foram ascite, fígado mais firme com cápsula brancacenta e edema de abomaso e mesentério. Os achados epidemiológicos e clinico-patológicos levantados podem ser visualizados no Quadro 1.

Foram realizadas biópsias de fígado de bovinos em nove propriedades $(3,5,7,8,10,11,13,15$ e 16$)$. De um total de 3.859 bovinos, 88 foram submetidos ao procedimento (Fig.2) e desses, 69 (78,4\%) foram positivos e 19 (21,59\%) negativos para intoxicação crônica por Senecio. Microscopicamente, as lesões foram caracterizadas por fibrose periportal ( $78,4 \%$ dos casos), megalocitose $(76,13 \%$ dos casos) e proliferação de ductos biliares $(68,13 \%$ dos casos). Essas lesões variaram entre discreta e acentuada, especialmente quanto ao grau de fibrose. Nos casos acentuados observou- -se estase biliar. Dos casos positivos para seneciose crônica, $64(92,75 \%)$ apresentaram megalocitose, $56(81,16 \%)$ hiperplasia de ductos biliares e $67(97,10 \%)$ fibrose periportal. Em dois casos positivos, a fibrose foi o único achado e a classificação foi baseada na morfologia da lesão que variou de discreta a moderada. Dos casos negativos, dois apresentaram megalocitose leve e dois com fibrose discreta. A bilestase foi observada no fígado de apenas três bovinos (Quadro 2).

\section{DISCUSSÃO}

A região em estudo é caracterizada, na sua maioria, por grandes propriedades com pastagem nativa e rebanhos criados de forma extensiva, além de apresentar períodos

Quadro 2. Achados microscópicos em fígados de bovinos com suspeita de intoxicação por Senecio spp. submetidos a biópsia hepática em propriedades do Oeste do Rio Grande do Sul de 2011 a 2014

\begin{tabular}{|c|c|c|c|c|c|c|c|c|c|c|c|c|c|}
\hline \multirow{2}{*}{$\begin{array}{l}\text { Proprie- } \\
\text { dade } \\
\text { (№ de } \\
\text { biópsias) }\end{array}$} & \multirow{2}{*}{ Município } & \multicolumn{5}{|c|}{ Achados microscópicos no fígado } & \multirow{2}{*}{$\begin{array}{c}\text { Proprie- } \\
\text { dade } \\
\text { (№ de } \\
\text { biópsias) } \\
\end{array}$} & \multirow{2}{*}{ Município } & \multicolumn{5}{|c|}{ Achados microscópicos no fígado } \\
\hline & & $\begin{array}{c}\text { Fibrose } \\
\text { peri- } \\
\text { portal }\end{array}$ & Megalocitose $\mathrm{H}$ & $\begin{array}{l}\text { Hiperplasia } \\
\text { de ductos } \\
\text { biliares }\end{array}$ & ilestase & Conclusão & & & $\begin{array}{c}\text { Fibrose } \\
\text { peri- } \\
\text { portal }\end{array}$ & Megalocitose & $\begin{array}{l}\text { Hiperplasia } \\
\text { de ductos } \\
\text { biliares }\end{array}$ & ilestase & Conclusão \\
\hline \multirow[t]{4}{*}{$3(4)$} & Alegrete & - & - & - & - & Negativa & $8(19)$ & Maçambará & +++ & +++ & +++ & - & $\mathrm{P}$ \\
\hline & & + & - & - & - & $\mathrm{P}^{\mathrm{a}}$ & & & +++ & ++ & ++ & - & $\mathrm{P}$ \\
\hline & & +++ & - & - & - & $\mathrm{P}$ & & & ++ & ++ & + & - & $\mathrm{P}$ \\
\hline & & - & - & - & - & Negativa & & & - & ++ & - & - & Negativa \\
\hline \multirow[t]{7}{*}{$5(7)$} & Uruguaiana & ++ & ++ & ++ & - & $\mathrm{P}$ & & & ++ & ++ & ++ & - & $\mathrm{P}$ \\
\hline & & ++ & ++ & ++ & - & $\mathrm{P}$ & & & ++ & ++ & ++ & - & $\mathrm{P}$ \\
\hline & & - & - & - & - & Negativa & & & ++ & + & ++ & - & $\mathrm{P}$ \\
\hline & & + & + & + & - & $\mathrm{P}$ & & & +++ & +++ & +++ & - & $\mathrm{P}$ \\
\hline & & ++ & ++ & - & - & $\mathrm{P}$ & & & ++ & - & ++ & - & $\mathrm{P}$ \\
\hline & & +++ & +++ & +++ & - & $\mathrm{P}$ & & & ++ & - & ++ & + & $\mathrm{P}$ \\
\hline & & +++ & ++ & ++ & - & $\mathrm{P}$ & & & + & + & + & & $\mathrm{P}$ \\
\hline \multirow[t]{28}{*}{7 (28) } & Quaraí & ++ & ++ & ++ & - & $\mathrm{P}$ & & & - & - & - & - & Negativa \\
\hline & & - & - & - & - & Negativa & & & + & + & + & - & $\mathrm{P}$ \\
\hline & & ++ & + & ++ & - & $\mathrm{P}$ & & & + & + & + & - & $\mathrm{P}$ \\
\hline & & ++ & ++ & ++ & - & $\mathrm{P}$ & $10(2)$ & Maçambará & + & + & + & - & $\mathrm{P}$ \\
\hline & & - & - & - & - & Negativa & & & ++ & + & + & - & $\mathrm{P}$ \\
\hline & & - & - & - & - & Negativa & $11(5)$ & São Borja & ++ & ++ & - & - & $\mathrm{P}$ \\
\hline & & ++ & ++ & - & - & $\mathrm{P}$ & & & + & + & - & - & $\mathrm{P}$ \\
\hline & & ++ & ++ & ++ & - & $\mathrm{P}$ & & & + & - & - & - & Negativa \\
\hline & & ++ & ++ & ++ & - & $\mathrm{P}$ & & & - & - & - & - & Negativa \\
\hline & & - & - & - & - & Negativa & & & - & ++ & - & - & Negativa \\
\hline & & ++ & ++ & ++ & - & $P$ & $13(1)$ & Uruguaiana & +++ & +++ & +++ & - & P \\
\hline & & +++ & +++ & +++ & - & $\mathrm{P}$ & $15(9)$ & Maçambará & ++++ & ++++ & +++ & - & $\mathrm{P}$ \\
\hline & & +++ & +++ & ++ & - & $\mathrm{P}$ & & & +++ & +++ & +++ & - & $\mathrm{P}$ \\
\hline & & - & - & - & - & Negativa & & & +++ & ++++ & ++++ & - & $\mathrm{P}$ \\
\hline & & - & ++ & ++ & - & $\mathrm{P}$ & & & +++ & ++++ & ++++ & - & $\mathrm{P}$ \\
\hline & & ++ & ++ & ++ & - & $\mathrm{P}$ & & & +++ & +++ & ++++ & - & $\mathrm{P}$ \\
\hline & & +++ & ++ & ++ & - & $\mathrm{P}$ & & & +++ & ++++ & ++++ & - & $\mathrm{P}$ \\
\hline & & ++ & ++ & - & - & $\mathrm{P}$ & & & + & - & - & - & Negativa \\
\hline & & + & + & - & - & $\mathrm{P}$ & & & ++ & ++ & ++ & - & $\mathrm{P}$ \\
\hline & & - & - & - & - & Negativa & & & ++ & ++++ & ++++ & - & $\mathrm{P}$ \\
\hline & & ++ & ++ & ++ & ++ & $\mathrm{P}$ & $16(13)$ & Maçambará & ++ & +++ & +++ & - & $\mathrm{P}$ \\
\hline & & ++ & ++ & ++ & ++ & $\mathrm{P}$ & & & + & ++ & ++ & - & $\mathrm{P}$ \\
\hline & & ++ & ++ & ++ & - & $\mathrm{P}$ & & & ++ & +++ & +++ & - & $\mathrm{P}$ \\
\hline & & - & - & - & - & Negativa & & & - & - & - & - & Negativa \\
\hline & & +++ & +++ & +++ & - & $\mathrm{P}$ & & & +++ & ++++ & ++++ & - & $\mathrm{P}$ \\
\hline & & ++ & ++ & ++ & - & $\mathrm{P}$ & & & +++ & +++ & +++ & - & $\mathrm{P}$ \\
\hline & & ++ & ++ & ++ & - & $\mathrm{P}$ & & & +++ & ++++ & ++++ & - & $\mathrm{P}$ \\
\hline & & - & - & - & - & Negativa & & & +++ & ++++ & +++ & - & $\mathrm{P}$ \\
\hline \multirow[t]{5}{*}{$8(19)$} & Maçambará & - & - & - & - & Negativa & & & +++ & ++++ & ++++ & - & $\mathrm{P}$ \\
\hline & & - & - & - & - & Negativa & & & +++ & ++++ & ++++ & - & $\mathrm{P}$ \\
\hline & & ++ & ++ & - & - & $\mathrm{P}$ & & & ++++ & ++++ & ++++ & - & $\mathrm{P}$ \\
\hline & & ++ & + & + & - & $\mathrm{P}$ & & & +++ & + & + & - & $\mathrm{P}$ \\
\hline & & ++ & ++ & - & - & $\mathrm{P}$ & & & +++ & ++++ & ++++ & - & $\mathrm{P}$ \\
\hline
\end{tabular}

$\overline{{ }^{a} \text { Bovinos positivos para seneciose: + discreta, ++ leve, }+++ \text { moderado }},++++$ acentuado, - sem alteração. 
de estiagem com elevadas temperaturas (máxima $=40^{\circ} \mathrm{C}$ ) e invernos rigorosos com temperaturas baixas, por vezes negativas, e com grande escassez de pastagens. Tais fatores favorecem a intoxicação de bovinos por Senecio, e mesmo que a planta apresente baixa palatabilidade o seu consumo é elevado quando há escassez de pastagens (Driemeier et al. 1991, Barros et al. 1992, Karam et al. 2002, Grecco et al. 2011, Tokarnia et al. 2012).

Nos períodos do ano caracterizados por grande oscilação de temperatura ocorre aumento das exigências metabólicas dos bovinos (Peterson \& Culvenor 1983, Karam et al. 2004), fator que pode contribuir para a maior ocorrência da intoxicação de bovinos nessa região. Na maioria dos casos desse estudo, os bovinos apresentaram sinais clínicos no final do inverno e durante o verão, aspecto semelhante ao descrito em estudos realizados nas regiões central e sul do Rio Grande do Sul (Barros et al. 1987, Méndez 1993, Karam et al. 2004, Grecco et al. 2010, Karam et al. 2011). Esses dois períodos exigem dos bovinos uma grande atividade metabólica, pois correspondem, respectivamente, a períodos de escassez de forragem, pelas baixas temperaturas e ao terço final da gestação da maioria das vacas dos rebanhos (inverno), bem como as elevadas temperaturas (verão), quando a exigência metabólica é maior e tradicionalmente ocorre a estação de monta com cobertura das vacas (Cardoso et al. 2000).

Atualmente, é proposto que a menor ocorrência da seneciose em bovinos machos jovens ocorre por menor ingestão da planta quando comparado com as vacas (Karam et al. 2004). No entanto, como proposto em outros estudos (Barros et al. 2007) e também observado nos casos aqui abordados, a permanência dos animais em contato com a planta por poucos dias ou semanas seria suficiente para desencadear a intoxicação também nos machos jovens após o desmame, uma vez que, a maioria dos rebanhos possui grande número de animais nessa faixa etária, destinados a engorda e abate. Nesse estudo foi observado que, diferentemente das fêmeas, os machos sofrem menor estresse, pois as melhores pastagens são destinadas a esse grupo na maioria das vezes para propiciar melhor ganho de peso. Possivelmente, a menor ocorrência da seneciose em terneiros esteja mais relacionada com as menores mudanças no manejo e, consequentemente, menos estresse metabólico (Prakash et al. 1999).

Durante as visitas às propriedades rurais se observou mais frequentemente Senecio brasiliensis e S. heterotrichius. Essa última espécie, assim como $S$. selloi, S. oxyphyllus e S. leptolobus apresentam morfologia bastante distinta de S. brasiliensis, especialmente nas fases de brotação (sem flores), e não foram reconhecidos pela maioria dos produtores e técnicos como plantas do gênero Senecio ("maria-mole"). Esse aspecto chama a atenção para a possibilidade de que muitos surtos podem não ter sido diagnosticados. Deve ser ressaltado que, na maioria das propriedades, os campos eram intensamente invadidos por Senecio, muitas vezes, pequeno e já com sinais de pastejo em meio a grande quantidade de capim-annoni (Eragrostis plana) sem qualquer tipo de manejo, o que o torna esse capim menos palatável, fibroso, mais seco e com baixo potencial nutricional
(Reis e Coelho 2000, Carlotto et al. 2010). Nessas condições, o consumo de Senecio pode ser favorecido, visto que é uma das poucas plantas que se mantêm verdes durante o inverno.

Os sinais clínicos descritos para os bovinos desse estudo são compatíveis com seneciose crônica (Riet-Correa et al. 2007, Grecco et al. 2010). No entanto, o desconhecimento das principais características da doença e a dificuldade na remessa de material para diagnóstico, por parte dos técnicos nas propriedades, ainda permite que muitos casos de seneciose não sejam confirmados. Esses aspectos confirmam a suspeita de que os índices de morbidade ainda são subestimados e que os prejuízos causados pela intoxicação podem ser ainda mais altos (Riet-Correa \& Medeiros 2001).

Foi observado no estudo que, na maioria das vezes, os bovinos com suspeita da intoxicação que estavam mais magros eram mantidos na propriedade por mais tempo com o intuito de melhorar sua condição corporal o que provavelmente elevam os prejuízos. Isso ocorre, pois na maioria dos casos os bovinos com algum grau de lesão hepática tendem a desenvolver o quadro mais grave que geralmente culmina com a morte ou eutanásia. No presente estudo, foram realizadas necropsias de bovinos, por médicos veterinários nas propriedades e os achados macroscópicos predominantes foram ascite, fígado mais firme com cápsula brancacenta e edema de abomaso e mesentério. Esses achados caracterizam a forma crônica da intoxicação (Lucena et al. 2010). No entanto, nenhuma amostra de tecido foi encaminhada para avaliação histopatológica, especialmente por dificuldade logística no encaminhamento de amostras, após a realização das necropsias.

A técnica de biópsia hepática realizada durante o estudo em bovinos de nove propriedades se mostrou de fácil execução e avaliada positivamente pelos médicos veterinários. A biópsia hepática é um excelente método de diagnóstico para a seneciose e permite estabelecer a real situação dos rebanhos frente à ocorrência da intoxicação (Barros et al. 2007, Tokarnia et al. 2012). 0 procedimento auxiliou sobremaneira a confirmação do diagnóstico e a seleção mais criteriosa dos bovinos a serem descartados dos rebanhos.

Após a confirmação da intoxicação nos bovinos, os proprietários foram instruídos a realizar o controle da planta com a inserção de ovinos nas áreas de pastoreio, na proporção de um ovino para cada dois hectares, especialmente durante os meses de inverno, quando tem início a brotação da planta (Driemeier et al. 1991, Barros et al. 1992, Soares et al. 2000, Karam et al. 2004, Grecco et al. 2011, Karam et al. 2013). 0 pastoreio dos ovinos no inverno interfere drasticamente no desenvolvimento da planta e diminui a difusão das sementes nas pastagens (Karam et al. 2013). Em estudos na região sul do Rio Grande do Sul, observou-se maior ocorrência de surtos quando houve redução da população de ovinos (Karam et al. 2004). Essa diminuição do rebanho ovino nos últimos anos também é percebida na microrregião da Campanha Ocidental (IBGE, 2012) que integra o oeste do RS e, segundo os produtores está relacionada com a desvalorização da lã, com as perdas por parasitoses, dificuldade com o manejo de forma extensiva e o abigeato. 
Foi relatado por diversos produtores o baixo índice reprodutivo de lotes de vacas, especialmente em anos de maior ocorrência de Senecio, mesmo em propriedades com acompanhamento técnico periódico e alta tecnologia envolvida no processo. Na maior parte dos casos permanecia desconhecida a causa do declínio da qualidade reprodutiva desses rebanhos. Recentemente, um estudo na região identificou alterações gonadais em vacas com a forma crônica de intoxicação por Senecio (Krabbe et al. 2015). Embora diversas condições patológicas possam desencadear déficit reprodutivo em bovinos, em muitos casos a etiologia não é determinada (Grunert et al. 2005, Riet-Correa et al. 2007) e a intoxicação por Senecio deve ser investigada também como possível causa para esses déficits.

Agradecimentos.- À FAPERGS e ao CNPq pelo auxílio financeiro.

\section{REFERÊNCIAS}

Anjos B.L., Nobre V.M.T., Dantas A.F.M., Medeiros R.M.T., Oliveira Neto T.S., Molyneux R.J. \& Riet-Correa F. 2010. Poisoning of sheep by seeds of Crotalaria retusa: acquired resistance by continuous administration of low doses. Toxicon 55:28-32.

Anjos B.L. 2014. Comunicação pessoal (Universidade Federal do Pampa, RS).

Barros C.S.L., Metzdorf L.L. \& Peixoto P.V. 1987. Ocorrência de surtos da intoxicação por Senecio brasiliensis (Compositae) em bovinos no Rio Grande Sul. Pesq. Vet. Bras. 7(4):101-107.

Barros C.S.L., Driemeier D., Pilati C., Barros S.S. \& Castilhos L.M.L. 1992. Senecio spp. poisoning in cattle in southern Brazil. Vet. Hum. Toxicol. 34(3):241-246.

Barros C.S.L., Castilhos L.M.L., Rissi D.R., Krommers G.D. \& Rech R.R. 2007. Biópsia hepática no diagnóstico da intoxicação por Senecio brasiliensis (Asteraceae) em bovinos. Pesq. Vet. Bras. 27(1):53-60.

Braga M.M., Castilhos L.M.L. \& Santos M.N. 1985. Biópsia hepática em bovinos: proposta de uma nova técnica. Revta Centro Ciênc. Rurais, Santa Maria, 15:79-88.

Cardoso F.F., Cardellino R.A. \& Campos L.T. 2000. Época de nascimento no crescimento de bezerros Aberdeen Angus criados no Rio Grande do Sul e suas implicações no melhoramento genético. Ciência Rural 30(6):1047-1051.

Carlotto S.B., Medeiros R.B., Pelegrini C.B., Garcia R.P.A., Lisboa C.A.V. \& Saibro J.C. 2010. Comportamento ingestivo diurno de vacas primíparas em pastagem nativa dominada por capim-annoni-2 com suplementação proteica e mineral em diversas estações climáticas. Revta Bras. Zootec. 39(3):454-461.

Driemeier D., Barros C.S.L. \& Pilati C. 1991. Seneciose em bovinos. Hora Vet. 59:23-30.

Grecco F.B., Shild A.L., Estima-Silva P., Marcolongo-Pereira C., Soares M.P. \& Salles E.S.V. 2010. Aspectos epidemiológicos e padrões das lesões hepáticas em 35 surtos de intoxicação por Senecio spp. em bovinos no sul do Rio Grande do Sul. Pesq. Vet. Bras. 30(5):389-397.

Grecco F.B., Estima-Silva P., Marcolongo-Pereira C., Soares M.P., Collares G. \& Schild A.L. 2011. Seneciose crônica em ovinos no sul do Rio Grande do Sul. Pesq. Vet. Bras. 31(4):326-330.

Grunert E., Birgel L.M. \& Vale W.G. 2005. Clínica da reprodução dos animais mamíferos domésticos. Varela, São Paulo. 502p.
IBGE 2012. Pecuária Rebanho Bovino e Ovino. Instituto Brasileiro de Geografia e Estatística. Disponível em <https://cidades.ibge.gov.br/xtras/ home.php> Acesso em 1 mai. 2017.

Karam F.S.C., Méndez M.C., Jarenkow J.A. \& Riet-Correa F. 2002. Fenologia de quatro espécies tóxicas de Senecio (Asteraceae) na região Sul do Rio Grande do Sul. Pesq. Vet. Bras. 22(1):33-39.

Karam F.S.C., Soares M.P., Haraguchi M., Riet-Correa F., Méndez M.C. \& Jarenkow J.A. 2004. Aspectos epidemiológicos da seneciose na região sul do Rio Grande do Sul. Pesq. Vet. Bras. 24(4):191-198.

Karam F.C., Schild A.L. \& Mello J.R.B. 2011. Intoxicação por Senecio spp. em bovinos no Rio Grande do Sul: condições ambientais favoráveis e medidas de controle. Pesq. Vet. Bras. 31(7):603-609.

Karam F.C., Moraes J.C.F. \& Schild A.L. 2013. Controle de Senecio spp. com pastoreio ovino de acordo com sua infestação e fenologia. Pesq. Vet. Bras. 33(9):1109-1115.

Krabbe A., Gonçalves M.A., Pozzobon R., Pessoa C.R.M., Costa R.A. \& Anjos B.L. 2015. Megalocitose de células luteínicas grandes de vacas prenhes com seneciose crônica. Pesq. Vet. Bras. 35(1):33-38.

Lucena R.B., Rissi D.R., Maia L.A., Flores M.M., Dantas A.F.M., Nobre V.M.T., Riet-Correa F. \& Barros C.S.L. 2010. Intoxicação por alcalóides pirrolizidínicos em ruminantes e equinos no Brasil. Pesq. Vet. Bras. 30(5):447452.

Méndez M.C. 1993. Intoxicação por Senecio spp., p.43-57. In: Riet-Correa F., Méndez M.C. \& Schild A.L. (Eds), Intoxicações por Plantas e Micotoxicoses em Animais Domésticos. Agropecuaria Hemisferio Sur, Montevideo.

Pessoa C.R.M., Medeiros R.M.T. \& Riet-Correa F. 2013. Importância econômica, epidemiologia e controle das intoxicações por plantas no Brasil. Pesq. Vet. Bras. 33(6):752-758.

Peterson J.E. \& Culvenor C.C.J. 1983. Hepatotoxic pyrrolizidine alkaloids, p.637-671. In: Keeler R.F. \& Tu A.T. (Eds), Handbook of Natural Toxins. Vol.1. Marcel Dekker, New York.

Prakash A.S., Perera T.N., Reilly P.E.B. \& Seawright A.A. 1999. Pirrolizidine alkaloids in human diet. Mutat. Res. 443:53-67.

Reis J.C.L. \& Coelho R.W. 2000. Controle do capim-annoni-2 em campos naturais e pastagens. Circular Técnica 22, Embrapa Clima Temperado, Pelotas. 21p.

Riet-Correa F. \& Medeiros R.M.T. 2001. Intoxicações por plantas em ruminantes no Brasil e no Uruguai: importância econômica, controle e riscos para a saúde pública. Pesq. Vet. Bras. 21:38-42.

Riet-Correa F., Schild A.L., Lemos R.A.A. \& Borges J.R.J. 2007. Doenças de Ruminantes e Equinos. Vol.1. 3a ed. Pallotti, Santa Maria. 722p.

Rissi D.R., Rech R.R., Pierezan F., Gabriel A.L., Trost M.E., Brum J.S., Kommers G.D. \& Barros C.S.L. 2007. Intoxicações por plantas e micotoxinas associadas a plantas em bovinos no Rio Grande do Sul: 461 casos. Pesq. Vet. Bras. 27(7):261-268.

Schild A.L., Motta A.C., Riet-Correa F., Karam F.C. \& Grecco F.B. 2004. Photosensitivity in cattle in southern Brazil, p.162-166. In: Acamovic T., Stewart C.S. \& Pennycott T.W. (Eds), Poisoning Plants and Related Toxins. CAB International, London.

Soares M.P., Riet-Correa F., Méndez M.C., Rosa F.G. \& Carreira E.G. 2000. Controle biológico de Senecio spp. com pastoreio de ovinos. Anais II Reunión Argentina de Patologia Veterinária, Faculdad de Ciencias Veterinarias, Universidad Nacional del Nordeste, Corrientes, Argentina, p.79. (Resumo)

Tokarnia C.H., Brito M.F., Barbosa J.D., Peixoto P.V. \& Döbereiner J. 2012. Senecio spp., p.177-191. In: Ibid. (Eds), Plantas Tóxicas do Brasil para Animais de Produção. $2^{\underline{a}}$ ed. Editora Helianthus, Rio de Janeiro. 